International Journal of Engineering \& Technology, $7(2.5)(2018) 77-83$
International Journal of Engineering \& Technology
WPC
Website: www.sciencepubco.com/index.php/IJET
Research Paper

\title{
Iris Segmentation
}

\author{
Anis Farihan Mat Raffei ${ }^{1}$, Rohayanti Hassan ${ }^{1 *}$, Shahreen Kasim², Hishamudin Asmuni ${ }^{1}$, Asraful Syifaa' Ahmad ${ }^{1}$, \\ Rahmat Hidayat $^{3}$, Ansari Saleh Ahmar ${ }^{4,5}$ \\ ${ }^{1}$ Faculty of Computing, Universiti Teknologi Malaysia, Skudai, Johor, Malaysia \\ ${ }^{2}$ Faculty of Computer Science and Information Technology, Universiti Tun Hussein Onn Malaysia, Johor, Malaysia \\ ${ }^{3}$ Department of Information Technology, Politeknik Negeri Padang, Indonesia \\ ${ }^{4}$ Department of Statistics, Universitas Negeri Makassar, Makassar, Indonesia \\ ${ }^{5}$ AHMAR Institute, Makassar, Indonesia \\ *Corresponding author E-mail: rohayanti@utm.my
}

\begin{abstract}
The quality of eye image data become degraded particularly when the image is taken in the non-cooperative acquisition environment such as under visible wavelength illumination. Consequently, this environmental condition may lead to noisy eye images, incorrect localization of limbic and pupillary boundaries and eventually degrade the performance of iris recognition system. Hence, this study has compared several segmentation methods to address the abovementioned issues. The results show that Circular Hough transform method is the best segmentation method with the best overall accuracy, error rate and decidability index that more tolerant to 'noise' such as reflection.
\end{abstract}

Keywords: Iris recognition, Iris Segmentation

\section{Introduction}

Iris recognition system consists of several stages, and segmentation stage is the most crucial step. This stage provides measurement of iris area with desirability and precise for the subsequent stages which are the iris feature extraction and template matching stages. The current segmentation methods are able to localize the iris in cooperative environment. However, those methods still have limitation in localizing the iris especially in non-cooperative environment. In non-cooperative environment, an eye image is captured at the different distances and in the movement compared to the cooperative environment which captured the eye image at specific distance and at the static position [1]. This conditions have caused the eye image in non-cooperative environment has poor quality compared to cooperative environment. Thus, the existing methods lead to improper localizing the pupillary and limbic boundaries of iris and not tolerant to 'noise' such as reflections. According to Radman et al. [2], pupillary boundary is located between the iris and pupil regions while limbic boundary is located between the iris and sclera regions. The improper localizing the iris boundaries will lead to low recognition performance when high level of noise or less area of iris is extracted during the iris segmentation process. Occlusion of eyelids and eyelashes are also being removed in this stage to get the desired iris area.

\section{Related Works}

Circular integrodifferential operator has been popularly used by [3]-[5] to detect the iris boundaries. This operator defined the iris boundaries using three parameters; radius and center coordinate. It searches over the image domain using the blurred partial derivative maximum value to increase the normalized contour integral radius of image. This operator performs highly accurate in detecting the boundaries of iris in the cooperative environment [5], [6]. On the other hand, insufficient contrast between the iris and sclera and existence of high level of noise in an eye image has caused failure in detecting the limbic boundary. Least square curve fitting has been used by several researchers [7], [8] to localize the iris and eyelids boundaries. Li et al. [8] implemented this algorithm to determine the three parameters of the circular iris boundaries; center and radius, while $\mathrm{Li}$ et al. [9] used this algorithm to determine the edge points on the eyelids and applied it as parabolas. This algorithm gives better results in points' determination for cooperative database. Circular Hough transform based on Canny edge detection has been popularly used by a number of researchers [10]-[13] to localize the iris boundaries in cooperative and non-cooperative environments. It starts with determining the edge of iris boundaries with Canny edge detector and voting procedure to fit a circle to the boundary. This algorithm alone provided a good at extracting geometrical components from any given object and very tolerant of noise. Several researchers such as [14], [15] used combination of Hough transform and integrodifferential operator to localize the iris. This combined method showed good performance for the iris segmentation of cooperative database. However, the performance of this combined method might reduce if it is tested using noncooperative database. For the upper and lower eyelids occlusion, Daugman modeled them as parabolic arcs of integrodifferential operator [6], [16], [17]. The similar operator is also used to localize eyelids' border, with applying the search contour from circular to curvature.

\section{Material and Methods}

This section gives details explanation and examples of the selected database and techniques used in this research. 


\subsection{Frontal Visible Eye Images}

There are many publicly databases available for iris recognition studies and two wavelengths were used to capture the eye images: near infrared and visible. This research chose visible wavelength of eye images rather than near infrared wavelength because this wavelength is safer than near infrared wavelengths which according to Proenca [18], excessive level of near infrared wavelength can danger the eye. Examples of visible light databases include the University of Olomuc (UPOL: Demirel and Anbarjafari [19]) and version two of the University of Beira Interior (UBIRISv2: Proenca et al., 2010). The UBIRISv2 database was the preferred database and it was selected for this research. This is because the eye images in this database were captured in non-cooperative environment (at different distances) whiles the UPOL databases were captured in cooperative environment (at rigid position). Moreover, this database contained high level of realistic noise such as blurring, contact lenses, reflections, occlusion of hair and occlusion of glasses compared to the cooperative database which contained less 'noisy' effects. The eye images in this database were obtained from 261 subjects with most of them was captured in two sessions. Each session captured 15 eye images for each (left and right) eye at distance of four to eight meters. Table 1 gives several examples of eye images that were used in this research in which approximately 1,000 frontal eye images were randomly chosen and were divided according to distances at which they were captured.

\begin{tabular}{|c|c|c|c|c|c|}
\hline \multirow{2}{*}{ Type } & \multicolumn{5}{|c|}{ Distance (meter) } \\
\hline & 4 & 5 & 6 & 7 & 8 \\
\hline Example of eye imag & & $y=-$ & & & \\
\hline Total eye images & 200 & 200 & 200 & 200 & 200 \\
\hline
\end{tabular}

\subsection{Segmentation of Iris}

Segmentation stage is very important process in order to get the desired iris area which to be used for the subsequent stages; iris feature extraction and template matching. This research used circular Hough transform which believed are able to properly localize the limbic and pupillary boundaries of iris which was captured under visible light and in non-cooperative environment To get the desired iris area, several steps need to execute where firstly, the eye image is formed into binarized edge by using Canny edge detector. Then, circular Hough transform is implemented to obtain the limbic and pupillary boundaries of iris. The segmentation process ends with the eyelid and eyelashes removal using linear Hough transform and thresholding algorithm. The segmentation process started with limbic and followed by pupillary boundaries. Each of the process stated is explained in the following subsections.

\subsubsection{Canny Edge Detection}

There are several edge detections such as Sobel, Laplacian of Gaussian, Prewitt and Robert's operator. The edge detection is important tool to characterize the lines and was used by many researchers from the various fields [20]-[22]. They discovered that the Canny edge detection provided better result in detecting the lines compared to the other edge tools. Thus, this research used the Canny detector to detect the iris boundaries. There are several processes for Canny edge detector. Firstly, a visible eye image is converted into grayscale intensity. Then, it is smoothed using Gaussian filter to blurring the eye image followed by gradients which were biased in the vertical direction for the limbic boundary. A line of limbic boundary can almost be observed in this step. Next, the hysteresis thresholding is used to create a binary map of eye image. Lastly, the limbic boundary of binarized eye image is detected using Canny edge detector. The similar process is performed for the pupillary boundary. The Canny detector will create an edge image at the end of the processes which will be used for the next step; circular Hough transform. Table 2 shows sample output images from the processes of Canny edge detection.

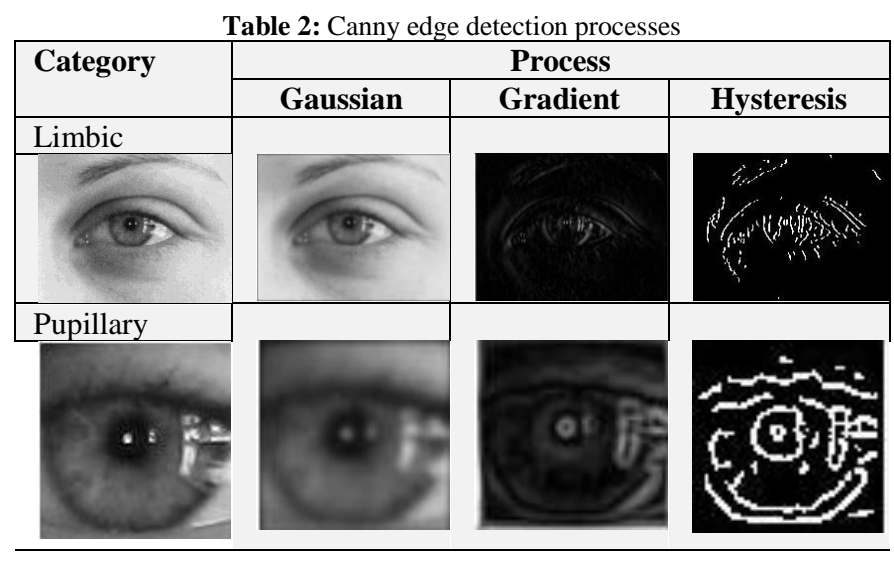

\subsubsection{Circular Hough Transform}

The binary edge map is then explored the votes on the circular Hough transform space to measure the three parameters of one circle which is $\left(h_{0}, k 0, R a d\right)$. A circular Hough transform space, $H T$ was formulated as shown below:

$$
H T\left(h_{0}, k_{0}, \operatorname{Rad}\right)=\sum_{n=1}^{j}\left(h_{n}, k_{n}, h_{0}, y_{0}, \operatorname{Rad}\right),
$$

where $\left(h_{n}, k_{n}\right)$ is a line pixel and $H T\left(h_{n}, k_{n}, h_{0}, k_{0}, R a d\right)$ is equal to 1 if $\left(h_{n}, k_{n}\right)$ is on the circle and 0 if otherwise. The location of ( $\left.h_{0}, k_{0}, R a d\right)$ with the maximum value of $H T\left(h_{0}, k_{0}, R a d\right)$ was selected as the parameter vector for the strongest circular boundary while $j$ is equal to 1 to $n$. This process starts with limbic boundary and followed by pupillary boundary of iris. Figure 1 showed the sample output of circular Hough transform for both limbic and pupillary boundaries of iris. Table 3 showed the output of localized iris at distance of four to eight meters. Although the eye image contained reflections, this method is still able to localize the iris boundaries properly. 


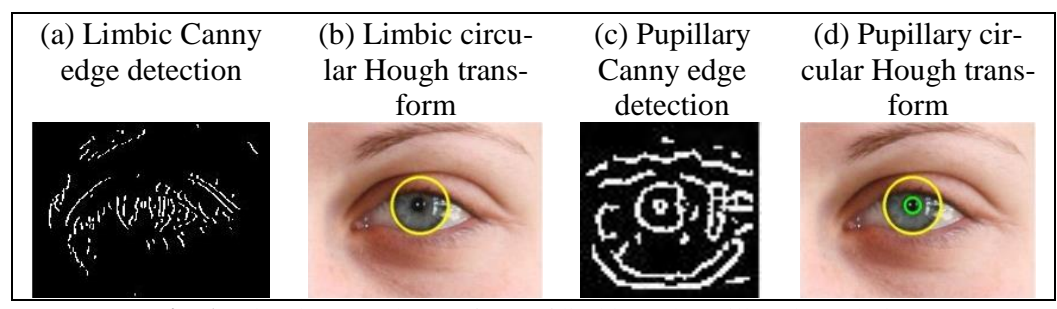

Fig. 1: Circular Hough transform of limbic and pupillary boundaries

Table 3: Circular Hough transform of iris for distance of four to eight meters

\begin{tabular}{|c|c|c|c|c|}
\hline \multirow{2}{*}{ Type } & \multicolumn{3}{|c|}{ Distance (meter) } \\
\cline { 2 - 5 } Example localized iris & $\mathbf{4}$ & $\mathbf{5}$ & $\mathbf{6}$ & $\mathbf{8}$ \\
\hline & & & \\
\hline
\end{tabular}

\subsubsection{Eyelids and Eyelashes Removal}

A linear Hough transform were used to detect the upper and lower eyelids. This method is superior to parabolic Hough transform it analyzes fewer parameters and requires less computational time [23], [24]. Other than that, a thresholding value was used (in this research, the thresholding value for the eyelashes was set to 20 pixels) to remove eyelashes from the eye images which if the length of eyelashes was lower than the thresholding value, it was considered to be an eyelash. Different iris databases have differ- ent thresholding value for eyelashes. This process started with upper eyelid and followed by lower eyelid. The overall process of iris segmentation is showed in Figure 2 where the process starts with the limbic segmentation (processes of Canny edge detection and limbic circular Hough transform), then the pupillary segmentation (processes of pupillary Canny edge detection and pupillary circular Hough transform), followed by upper eyelids and eyelash removal, and lastly, lower eyelid and eyelash removal. Table 4 showed output of segmented iris with the eyelids and eyelashes removal for the eye images in distance of four to eight meters.
Original eye image

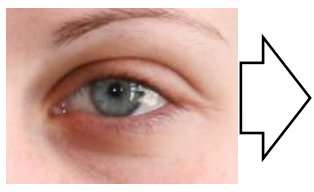

End result of iris segmentation

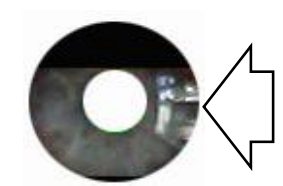

Limbic Canny edge detection
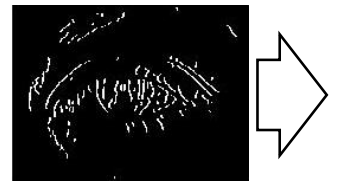

Lower eyelid and eyelash removal

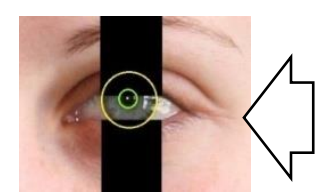

Limbic circular Hough transform

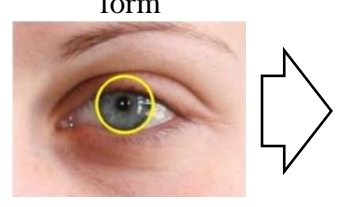

Upper eyelid and eyelash removal

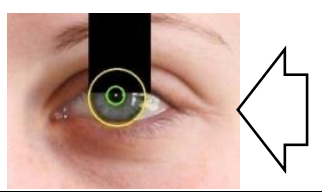

Pupillary Canny edge detection
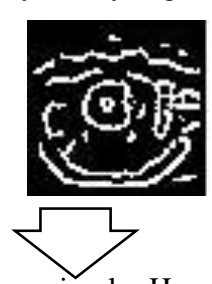

Pupillary circular Hough transform

Fig. 2: Overall process of iris segmentation

Table 4: Segmented iris of the eye images in distance of four to eight meters

\begin{tabular}{|c|c|c|c|c|c|}
\hline \multirow{2}{*}{ Type } & \multicolumn{3}{|c|}{ Distance (meter) } \\
\cline { 2 - 5 } Example of segmented iris & $\mathbf{4}$ & $\mathbf{5}$ & $\mathbf{6}$ & $\mathbf{8}$ \\
\hline & & &
\end{tabular}

\subsection{Normalization of Iris}

The next stage is to convert the circular segmented iris into a fixed rectangular dimension in order to allow extraction and comparisons in the subsequent stages. Figure 3 shows the mask normalization of an iris using a homogenous rubber sheet model where the white regions represent the noise created by eyelids and eyelashes. The model remaps each point within the iris region to a pair of polar coordinates $(r, \theta) ; r$ is on the interval $[0,1]$ and $\theta$ is angle $[0,2 \pi]$. The remapping of the iris region from $(\mathrm{x}, \mathrm{y})$ Carte- sian coordinates to the normalised non-concentric polar representation is modelled as

$$
I(x(r, \theta), y(r, \theta)) \rightarrow I(r, \theta),
$$

where $x(r, \theta)=(1-r) x p(\theta), y(r, \theta)=(1-r) y p(\theta)+r y l(\theta), I(x, y)$ is the iris region image, $(\mathrm{x}, \mathrm{y})$ are the original Cartesian coordinates, $(\mathrm{r}, \theta)$ are the corresponding normalized polar coordinates, (xp,yp) and $(\mathrm{xl}, \mathrm{yl})$ are the coordinates of the pupil and iris boundaries along the $\theta$ direction. The centre of the pupil acted as a reference point and the radial vectors passed through the iris. To form a pair of polar coordinate, several parameters were used to represent a rec- 
tangle which was $20 \times 240$ pixels. Table 5 showed sample results of normalized iris of eye images at distance of four to eight meters.

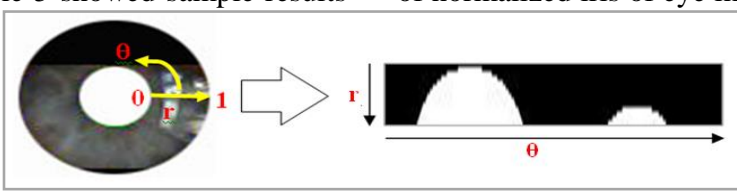

Fig. 3: Normalized iris of the eye images in distance of four to eight meters

Table 5: Normalized iris of eye images for distance of four to eight meters

\begin{tabular}{|c|c|c|c|c|c|}
\hline \multirow{2}{*}{ Type } & \multicolumn{3}{|c|}{ Distance (meter) } \\
\cline { 2 - 5 } Example of normalized iris & $\mathbf{4}$ & $\mathbf{5}$ & $\mathbf{6}$ \\
\hline
\end{tabular}

\subsection{Feature Extraction of Iris}

The process of feature extraction provides accuracy to the iris recognition by extracting the distinctive features of extracted iris. The distinctive features of iris were encrypted so that comparisons between templates could be made in template matching stage. A band pass decomposition of the iris image is employed by the majority of iris recognition systems in order to create an iris template. In this research, a one dimensional of log Gabor filter (formula 3) was used to obtain the local feature points of segmented iris in the Cartesian coordinate system. With the parameter determined during the normalization process, the number of bits used for the iris template in this research was set to 9,600 and the same number of bits was used for the mask template. The one dimensional of $\log$ Gabor filter, $L G$ was applied to each row of the normalized iris where $h_{0}$ is the center frequency and $\sigma$ is the bandwidth of the filter. Figure 4 showed example of feature templates of one dimensional log Gabor filter from normalized iris. Table 6 showed examples of feature templates obtained for the normalized iris in distance of four to eight meters.

$$
L G(h)=\exp \left(-\left(\log \left(h / h_{0}\right)\right)^{2} / 2\left(\log \left(\sigma / h_{0}\right)\right)^{2}\right)
$$
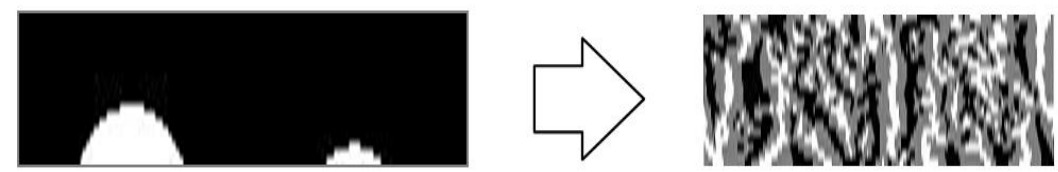

Fig. 4: Feature template of one dimensional log Gabor filter from normalized iris

Table 6: Feature templates of normalized iris for the distance of four to eight meters

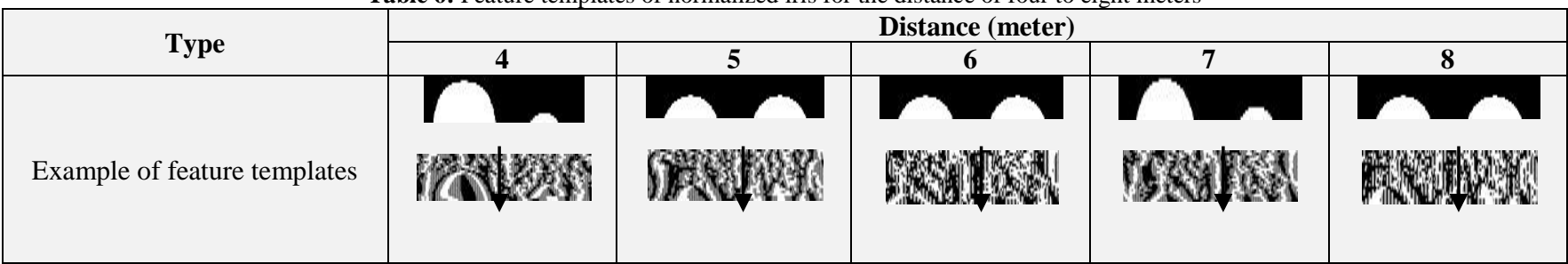

\subsection{Template Matching of Iris}

There are various templates matching methods such as the normalized correlation based matching [25] and Euclidean distance matching [26], [27] that can be used for pattern recognition. In this research, the hamming distance method, HD was performed to match between the two iris templates [28], [29]. This can be achieved in two steps. The first step is a logical operation where the XOR of the two binary vectors of length $k$ are determined. In the second step, the total number of one is determined. The similarity score or HD is measured by dividing the obtained sum by $k$. The hamming distance is formulated as follows:

$H D=\frac{1}{k} \sum_{i=1}^{k} P_{i} \oplus Q_{i}$

where $P$ and $Q$ are two templates from different iris images and $k$ is the total bits of those two templates. Each of the irises produces a different pattern of bit in a different area. The two iris codes produced by the same iris were parallel. The HD between the two patterns was equal to 0.5 if the two bits patterns were completely different because the difference between the patterns was completely random. The possibility of obtaining a score of 0.5 for any bit was either 1 or 0 . The possibility that the bits will agree or disagree is halfway between the two patterns. On the other hand, the HD will be close to 0 if the patterns were extracted from the same iris because the two bits patterns will be highly correlated.

\section{Results and Discussion}

This section gives the detailed explanation of results gained.

\subsection{Analysis of Iris Segmentation}

Table 7 shows results of accuracy of iris localization for limbic and pupillary boundaries using different methods. The accuracy of circular Hough transform method has the highest than the other 
methods: $86.1 \%$ for limbic and $70 \%$ for pupillary boundaries while the accuracy of integrodifferential operator method has the lowest for both boundaries: $66.5 \%$ for limbic and $57.8 \%$ for pupillary. The method of integrodifferential operator itself is not tolerant to 'noise' which is reflections; therefore, it incorrectly localized the iris boundaries. However, the combination of circular Hough transform and integrodifferential operator has improved the method of integrodifferential operator where $80.4 \%$ for limbic and $58.2 \%$ for pupillary localization. The accuracy of least square curve fitting method gives $80.1 \%$ for limbic and $58.2 \%$ for pupillary boundaries where the accuracy of pupillary boundary for this method is similar with the accuracy of pupillary boundary for the method of combination of circular Hough transform and integrodifferential operator. Although least square curve fitting algorithm is good in points determination, the existing of reflections in the iris has disrupted the determination between points of iris boundaries and points of reflections. The method of circular Hough transform gives better performance in localizing the iris However, the existing of large reflection in iris has caused incorrect localization of pupillary boundaries. This is because this method was falsely detected the edges of reflection as edges of pupillary. The limbic boundaries of iris were almost correctly localized by all methods because the large difference of region intensities between white sclera and iris has made the methods to properly localize the limbic boundary. Nevertheless, if the large reflection existed in the image, these methods could not localize the limbic boundary correctly. The output of limbic and pupillary localization for the different distances can be viewed in Table 8 where the existing of 'noise' which is large reflections has degraded the performance of iris localization methods especially for the pupillary boundary. Table 9 displays several outputs of incorrect localization of iris using different methods when the 'noise' which is reflections existed and was grouping into several categories.
Table 7: Accuracy of limbic and pupillary localization for different methods

\begin{tabular}{|l|c|c|}
\hline \multirow{2}{*}{\multicolumn{1}{|c|}{ Methods }} & \multicolumn{2}{|c|}{$\begin{array}{c}\text { Accuracy Localization } \\
\text { (\%) }\end{array}$} \\
\cline { 2 - 3 } & Limbic & Pupillary \\
\hline Circular Hough transform & 86.1 & 70.0 \\
\hline Integrodifferential Operator & 66.5 & 57.8 \\
\hline Least Square Curve Fitting & 80.1 & 58.2 \\
\hline $\begin{array}{l}\text { Circular Hough transform + In- } \\
\text { tegrodifferential Operator }\end{array}$ & 80.4 & 58.2 \\
\hline
\end{tabular}

\subsection{Accuracy of Iris Recognition}

Table 10 shows accuracy of iris recognition for different methods in distance of four to eight meters. The method of circular Hough transform has the highest accuracy compared to the other methods which $85.9 \%$ for four meter, $85.2 \%$ for five meter, $82.9 \%$ for six meter, $82.1 \%$ for both seven and eight meters. The method of integrodifferential operator has the lowest accuracy compared to the other methods which $69.0 \%$ for four meter, $67.1 \%$ for five meter, $66 \%$ for six meter, $62.1 \%$ for seven meter and $59.1 \%$ for eight meter. The combination of circular Hough transform and integrodifferential operator has increased the accuracy of integrodifferential operator which $80.1 \%$ for four meter, $79.9 \%$ for five meter, $75.9 \%$ for six meter, $73.4 \%$ for seven meter and $72.8 \%$ for eight meter. The accuracy of least square curve fitting method has almost the same with the method of combination of circular Hough transform and integrodifferential operator where $80.1 \%$ for four meter, $79 \%$ for five meter, $74.7 \%$ for six meter and $72.5 \%$ for both seven and eight meters. This can be concluded that as the distance increased, the accuracy of iris recognition for the different methods was decreased. This is because as the distance increased, the existing of 'noise' such as reflection has also increased. Thus, it caused incorrectly localization of iris boundaries especially the pupillary boundary as the existing of reflection made the methods falsely detect the edge of reflection as edge of pupillary boundary. The method of circular Hough transform that is tolerant to noise makes it correctly localize the iris boundaries.

Table 8: Localization of iris at distance of four to eight meters using different methods

\begin{tabular}{|c|c|c|c|c|c|}
\hline \multirow{2}{*}{ Methods } & \multicolumn{5}{|c|}{ Distance (meter) } \\
\hline & 4 & 5 & 6 & 7 & 8 \\
\hline \multicolumn{6}{|l|}{$\begin{array}{l}\text { Circular Hough Trans- } \\
\text { form }\end{array}$} \\
\hline \multicolumn{6}{|l|}{$\begin{array}{l}\text { Integrodifferential Op- } \\
\text { erator }\end{array}$} \\
\hline \multicolumn{6}{|l|}{$\begin{array}{l}\text { Least Square Curve } \\
\text { Fitting }\end{array}$} \\
\hline $\begin{array}{l}\text { Circular Hough Trans- } \\
\text { form + Integrodifferential } \\
\text { Operator }\end{array}$ & & & & & \\
\hline
\end{tabular}

Table 9: Categories of incorrect iris boundaries localization using different methods

\begin{tabular}{|c|c|c|c|c|}
\hline \multirow[b]{2}{*}{ Methods } & \multicolumn{4}{|c|}{ Category } \\
\hline & $\begin{array}{l}\text { Frontal eye with } \\
\text { off focus and } \\
\text { small reflection }\end{array}$ & $\begin{array}{c}\text { Frontal eye } \\
\text { image with large } \\
\text { reflection }\end{array}$ & $\begin{array}{c}\text { Frontal eye with spectacle and large } \\
\text { reflection }\end{array}$ & $\begin{array}{l}\text { Frontal eye with off } \\
\text { focus and large } \\
\text { reflection }\end{array}$ \\
\hline $\begin{array}{l}\text { Circular Hough } \\
\text { Transform }\end{array}$ & & & & \\
\hline
\end{tabular}




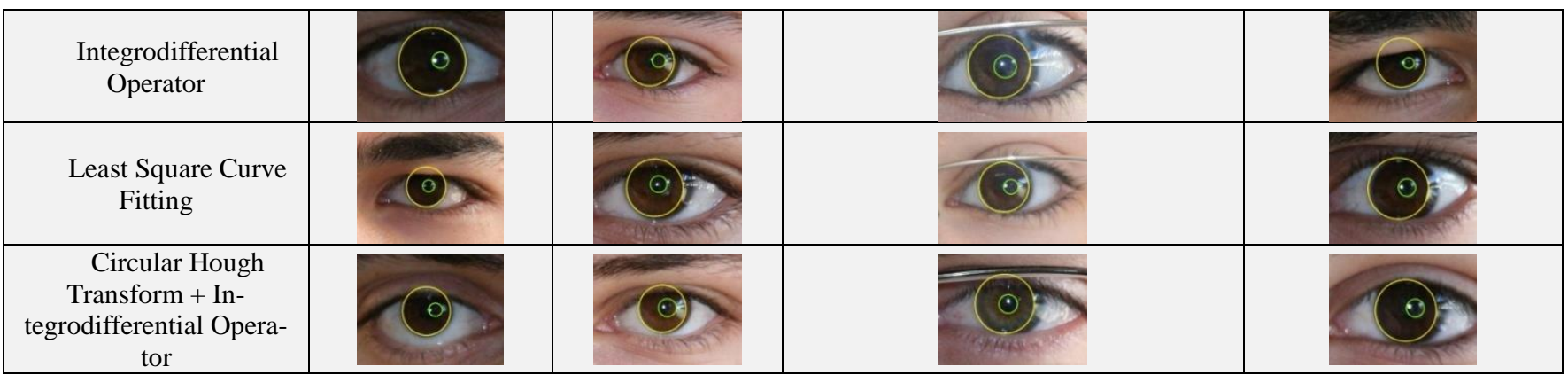

Table 10: Accuracy of iris recognition for different methods in distance of four to eight meters

\begin{tabular}{|l|c|c|c|c|c|c|}
\hline \multirow{2}{*}{ Methods } & \multicolumn{6}{c|}{ Accuracy (\%) } \\
\cline { 2 - 7 } & \multicolumn{5}{c|}{ Distance (meter) } \\
\cline { 2 - 7 } & $\mathbf{4}$ & $\mathbf{5}$ & $\mathbf{6}$ & $\mathbf{7}$ & $\mathbf{8}$ & Overall \\
\hline Circular Hough Transform & 85.9 & 85.2 & 82.9 & 82.1 & 82.1 & 85.1 \\
\hline Integrodifferential Operator & 69.0 & 67.1 & 66.0 & 62.1 & 59.1 & 66.7 \\
\hline Least Square Curve Fitting & 80.1 & 79.0 & 74.7 & 72.5 & 72.5 & 78.5 \\
\hline Circular Hough Transform + Integrodifferential Operator & 80.1 & 79.9 & 75.9 & 73.4 & 72.8 & 79.3 \\
\hline
\end{tabular}

\subsection{Equal error rate of Iris Recognition}

Table 11 shows results of equal error rate of iris recognition for different methods according to distance of four to eight meters. The method of circular Hough transform has the lowest percentage of equal error rate which $10.9 \%$ for four meter, $10.7 \%$ for five meter, $13.3 \%$ for six meter, $14.5 \%$ for seven meter and $15.6 \%$ for eight meter. The method of combination of circular Hough transform and integrodifferential operator has decreased the percentage of equal error rate for integrodifferential operator method where about $8.1 \%$ for four meter, $6.1 \%$ for five meter, $7.9 \%$ for six meter, $4.8 \%$ for seven meter and $8.8 \%$ for eight meter. The method of least square curve fitting has $15.9 \%$ for four meter, $18.4 \%$ for five meter, $19.5 \%$ for six meter and $23.9 \%$ for both seven and eight meters in percentages of equal error rate. Overall, the method of circular Hough transform have less than $15 \%$ in percentages of equal error rate while the methods of least square curve fitting, integrodifferential operator and combination of circular Hough transform and integrodifferential operator have more than $20 \%$. This can be concluded that correctly localize the iris will give better extraction of iris area where it is important to be used for the feature extraction and template matching process. If the iris is not correctly localize, less bit of features could be extracted during feature extraction process and thus, give high error identification during template matching process.

Table 11: Equal error rate of iris recognition for different methods in distance of four to eight meters

\begin{tabular}{|l|c|c|c|c|c|c|}
\hline \multirow{2}{*}{ Methods } & \multicolumn{5}{|c|}{ Equal error rate (\%) } \\
\cline { 2 - 7 } & & \multicolumn{5}{|c|}{ Distance (meter) } \\
\cline { 2 - 7 } & $\mathbf{4}$ & $\mathbf{5}$ & $\mathbf{6}$ & $\mathbf{7}$ & $\mathbf{8}$ & $\mathbf{O v e r a l l}$ \\
\hline Circular Hough Transform & 10.9 & 10.7 & 13.3 & 14.5 & 15.6 & 14.9 \\
\hline Integrodifferential Operator & 24.0 & 24.9 & 26.7 & 27.9 & 31.9 & 25.9 \\
\hline Least Square Curve Fitting & 15.9 & 18.4 & 19.5 & 23.9 & 23.9 & 21.6 \\
\hline Circular Hough Transform + Integrodifferential Operator & 15.9 & 18.8 & 18.8 & 23.1 & 23.1 & 20.9 \\
\hline
\end{tabular}

\subsection{Decidability of Iris Recognition}

Table 12 shows the output of decidability index of iris recognition for different methods in distance of four to eight meters. Decidability determines the separation distance between intra-class and inter-class distribution. The method of integrodifferential operator give lowest decidability index compared to the other methods where 2.10 for four meter, 2.05 for five meter, 1.99 for six meter, 1.80 for seven meter and 1.75 for eight meter. The combination of circular Hough transform and integrodifferential operator has increased the decidability index of integrodifferential operator where 2.21 for four meter, 2.18 for five and six meters, 2.10 for seven meter and 2.01 for eight meter. The method of circular Hough transform give highest decidability index which 2.60 for four meter, 2.57 for five meter, 2.55 for six meter and 2.39 for both seven and eight meters. The method of least square curve fitting has 2.20 for four meter, 2.17 for five meter, 2.14 for six meter and 2.07 for both seven and eight meters in decidability index. This can be concluded that the method of circular Hough transform provided good separation between inter-class and intraclass while the method of integrodifferential operator provided the least classes separation. A good separation of inter and intra-class give a good performance in identifying and verifying a person. The methods of least square curve fitting and combination of circular Hough transform and integrodifferential operator has almost the same performance in separating the inter and intra-class distributions.

Table 11: Decidability index of iris recognition for different methods in distance of four to eight meters

\begin{tabular}{|l|c|c|c|c|c|c|}
\hline \multirow{2}{*}{ Methods } & \multicolumn{5}{c|}{ Decidability Index } \\
\cline { 2 - 7 } & & \multicolumn{5}{c|}{ Distance (meter) } \\
\cline { 2 - 7 } & $\mathbf{4}$ & $\mathbf{5}$ & $\mathbf{6}$ & $\mathbf{7}$ & $\mathbf{8}$ & $\mathbf{O v e r a l l}$ \\
\hline Circular Hough Transform & 2.60 & 2.57 & 2.55 & 2.39 & 2.39 & 2.49 \\
\hline Integrodifferential Operator & 2.10 & 2.05 & 1.99 & 1.80 & 1.75 & 1.89 \\
\hline Least Square Curve Fitting & 2.20 & 2.17 & 2.14 & 2.07 & 2.07 & 2.09 \\
\hline Circular Hough Transform + Integrodifferential Operator & 2.21 & 2.18 & 2.18 & 2.10 & 2.01 & 2.15 \\
\hline
\end{tabular}




\section{Conclusion}

The method of circular Hough transform has provided a good localization of iris compared to the other methods: least square curve fitting, integrodifferential operator and combination of circular Hough transform and integrodifferential operator. This is because this method is very tolerant to 'noise' such as reflection which could detect the edge of reflections and iris boundaries correctly compared to the other methods. The combination of circular Hough transform and integrodifferential operator has improved the performance of integrodifferential operator method alone. Although the method of circular Hough transform provided good localization of iris, this method still have problem in localizing the pupillary boundary due to the existing of large reflection. Therefore, 'noise' such as large reflection that existed in the eye images especially in the iris area need to be removed so that the localization of limbic and pupillary boundaries can be performed correctly.

\section{Acknowledgement}

This research is funded by Research University Grant and Universiti Teknologi Malaysia under Vote No: 16H73.

\section{References}

[1] H. Proença, S. Filipe, R. Santos, J. Oliveira, and L. A. Alexandre, "The UBIRIS.v2: A database of visible wavelength iris images captured on-the-move and at-a-distance," IEEE Transactions on Pattern Analysis and Machine Intelligence, vol. 32, no. 8, pp. 1529-1535, 2010

[2] A. Radman, K. Jumari, and N. Zainal, "Iris segmentation: A review and research issues," in Communications in Computer and Information Science, 2011, vol. 179 CCIS, no. PART 1, pp. 698 708.

[3] Z. Zhou, P. Yao, Z. Zhuang, and J. Li, "A robust algorithm for iris localization based on radial symmetry and circular integro differential operator," in Proceedings of the 2011 6th IEEE Conference on Industrial Electronics and Applications, ICIEA 2011 2011, pp. 1742-1745.

[4] T. Tan, Z. He, and Z. Sun, "Efficient and robust segmentation of noisy iris images for non-cooperative iris recognition," Image and Vision Computing, vol. 28, no. 2, pp. 223-230, Feb. 2010

[5] M. Shamsi, P. B. Saad, S. B. Ibrahim, and A. R. Kenari, "Fast algorithm for iris localization using Daugman circular integro differential operator," in SoCPaR 2009 - Soft Computing and Pattern Recognition, 2009, pp. 393-398.

[6] X. Liu, P. Li, and Q. Song, "Eyelid localization in iris images captured in less constrained environment," in Lecture Notes in Computer Science (including subseries Lecture Notes in Artificial Intelligence and Lecture Notes in Bioinformatics), 2009, vol. 5558 LNCS, pp. 1140-1149.

[7] W. Li, M. Xie, and P. Yu, "A Fast and Robust Method for Iris Localization Using Light Spot," Journal of Computational Information Systems, vol. 7, no. 13, pp. 4619-4627, 2011.

[8] H. Li, Z. Sun, and T. Tan, "Accurate Iris Localization Using Contour Segments," International Conference on Pattern Recognition, no. Icpr, pp. 3398-3401, 2012.

[9] Y.-H. Li and M. Savvides, "An Automatic Iris Occlusion Estimation Method Based on High Dimensional Density Estimation," IEEE Transactions on Pattern Analysis and Machine Intelligence, vol. 35, no. 4, pp. 1-1, 2012

[10] A. Ghanizadeh, A. A. Abarghouei, S. Sinaie, P. Saad, and S. M. Shamsuddin, "Iris segmentation using an edge detector based on fuzzy sets theory and cellular learning automata," Applied Optics, vol. 50, no. 19, p. 3191, Jul. 2011.

[11] A. E. Yahya and M. J. Nordin, "Improving iris segmentation by specular reflections removable," in Proceedings 2010 International Symposium on Information Technology - Visual Informatics, ITSim'10, 2010, vol. 1, pp. 1-3.

[12] S. S. S, K. N. B. Murthy, S. Natarajan, M. R, and A. S, "Feed Forward Neural Network Based Eye Localization and Recognition Using Hough Transform," IJACSA International Journal of
Advanced Computer Science and Applications, vol. 2, no. 3, pp. 104-109, 2011

[13] Y. Chen, M. Adjouadi, C. Han, J. Wang, A. Barreto, N. Rishe, and J. Andrian, "A highly accurate and computationally efficient approach for unconstrained iris segmentation," Image and Vision Computing, vol. 28, no. 2, pp. 261-269, Feb. 2010.

[14] A. Bendale, A. Nigam, S. Prakash, and P. Gupta, "Iris segmentation using improved Hough transform," 8th International Conference on Emerging Intelligent Computing Technology and Applications, ICIC 2012, vol. 304 CCIS. Springer, Berlin, Heidelberg, pp. 408415, 2012.

[15] D. M. Rankin, B. W. Scotney, P. J. Morrow, D. R. McDowell, and B. K. Pierscionek, "Dynamic iris biometry: a technique for enhanced identification.," BMC research notes, vol. 3, no. 1, p. 182 Jul. 2010.

[16] N. K. Mahadeo and N. Bhattacharjee, "An efficient and accurate iris segmentation technique," in DICTA 2009 - Digital Image Computing: Techniques and Applications, 2009, pp. 347-352.

[17] M. Adam, F. Rossant, F. Amiel, B. Mikovicova, and T. Ea, "Reliable eyelid localization for iris recognition," in Lecture Notes in Computer Science (including subseries Lecture Notes in Artificial Intelligence and Lecture Notes in Bioinformatics), 2008 vol. 5259 LNCS, pp. 1062-1070.

[18] H. Proença, "Non-cooperative iris recognition: Issues and trends," in Signal Processing Conference, 2011.

[19] H. Demirel and G. Anbarjafari, "Iris recognition system using combined histogram statistics," 2008 23rd International Symposium on Computer and Information Sciences, pp. 1-4, Oct. 2008.

[20] B. Kaur, G. A. P. Anil, and E. M. M. E. In, "Comparative Study of Differentedge Detection Techniques," in International Journal of Engineering Science and Technology (IJEST), 2011, pp. 1927-1935.

[21] V. G. Narendra and K. S. Hareesh, "Study and comparison of various image edge detection techniques used in quality inspection and evaluation of agricultural and food products by computer vision," Int J Agric \& Biol Eng Open Access at Int J Agric \& Biol Eng, vol. 4, no. 2, pp. 83-90, 2011.

[22] M. Juneja and P. S. Sandhu, "Performance evaluation of edge detection techniques for images in spatial domain," International Journal of Computer Theory and Engineering, vol. 1, no. 5, pp. 614-622, 2009.

[23] T. H. Min and R. H. Park, "Eyelid and eyelash detection method in the normalized iris image using the parabolic Hough model and Otsu's thresholding method," Pattern Recognition Letters, vol. 30, no. 12, pp. 1138-1143, Sep. 2009.

[24] Y. K. Jang, B. J. Kang, and K. R. Park, "A study on eyelid localization considering image focus for iris recognition," Pattern Recognition Letters, vol. 29, no. 11, pp. 1698-1704, Aug. 2008.

[25] S. Dalal and T. Sahoo, "A Selective Feature Matching Approach for Iris Recognition," International Journal of Computer Applications, vol. 41, no. 20, pp. 975-8887, 2012.

[26] F. Sayeed, M. Hanmandlu, A. Q. Ansari, and S. Vasikarla, "Iris recognition using segmental Euclidean distances," in Proceedings 2011 8th International Conference on Information Technology: New Generations, ITNG 2011, 2010, pp. 520-525.

[27] L. Yu, D. Zhang, and K. Wang, "The relative distance of key point based iris recognition," Pattern Recognition, vol. 40, no. 2, pp 423-430, Feb. 2007.

[28] K. P. Hollingsworth, K. W. Bowyer, and P. J. Flynn, "Improved iris recognition through fusion of hamming distance and fragile bit distance," IEEE Transactions on Pattern Analysis and Machine Intelligence, vol. 33, no. 12, pp. 2465-2476, Dec. 2011

[29] R. P. Broussard, L. R. Kennell, R. W. Ives, and R. N. Rakvic, "An artificial neural network based matching metric for iris identification," 2008, vol. 6812, p. 68120S 